\title{
Nonparametric Liquefaction Triggering and Postliquefaction Deformations
}

\author{
J. S. Yazdi, M.ASCE${ }^{1}$; and R. E. S. Moss, Ph.D., P.E., M.ASCE ${ }^{2}$
}

\begin{abstract}
This study evaluates granular liquefaction triggering case-history data using a nonparametric approach. This approach assumes no functional form in the relationship between liquefied and nonliquefied cases as measured using cone penetration test (CPT) data. From a statistical perspective, this allows for an estimate of the threshold of liquefaction triggering unbiased by prior functional forms, and also provides a platform for testing existing published methods for accuracy and precision. The resulting threshold exhibits some unique trends, which are then interpreted based on postliquefaction deformation behavior. The range of postliquefaction deformations are differentiated into three zones: (1) large deformations associated with metastable conditions; (2) medium deformations associated with cyclic strain failure; and (3) small deformations associated with cyclic stress failure. Deformations are further defined based on the absence or presence of static driving shear stresses. This work presents a single simplified framework that provides quantitative guidance on triggering and qualitative guidance on deformation potential for quick assessment of risks associated with seismic soil liquefaction failure.
\end{abstract}

Author keywords: Liquefaction; Nonparametric; Triggering; Postliquefaction; Deformations.

\section{Introduction}

Seismic soil liquefaction is defined here as the response of saturated granular soils to strong ground shaking that results in elevated pore fluid pressure and reduced intergranular effective stress that often leads to moderate to high deformation potential. Because many of the key variables of soil liquefaction are disturbed when granular soil is sampled in situ, assessing liquefaction potential is relegated to assessing prior earthquake-induced field evidence of liquefied and complementary nonliquefied case histories. The simplified method was introduced by Seed and Idriss (1971) and the central concept has been used in practice since to predict if any given site is at risk for liquefaction triggering. Many methods since 1971 have built on the simplified method using various in situ testing techniques such as the standard penetration test (SPT), cone penetration test (CPT), Becker penetrometer (BPT), shear wave velocity measurements (VS), flat-plate dilatometer (DMT), and others. Because of the high repeatability, closely spaced measurements, ability to make multiple simultaneous measurements (e.g., $q_{c}, f_{s}, V_{s}$, and $k$ ), and the high-quality control of cone penetration testing, it is generally considered the most accurate and precise (Mayne et al. 2001). This study will focus on the cone penetration test (CPT) for the measurement of a soil's cyclic resistance (CRR) with the simplified method for the soils cyclic loading (CSR).

A nonparametric method is introduced in this study that estimates the relationship between case-history data of liquefied and nonliquefied sites. Nonparametric means no assumption is made

${ }^{1}$ Ph.D. Candidate, School of Natural and Built Environments, Univ. of South Australia, Mawson Lakes, SA 5095, Australia. E-mail: Javad .SadoghiYazdi@unisa.edu.au

${ }^{2}$ Professor, Dept. Civil and Environmental Engineering, California Polytechnic State Univ., San Luis Obispo, CA 93407 (corresponding author). E-mail: rmoss@calpoly.edu about the relationship of the threshold between these two data classes. Most existing methods in practice assume some functional relationship prior to evaluating the fit to the data, which implies some prior knowledge about this relationship. This nonparametric method provides a statistically unbiased fitting estimate of the threshold and also provides a framework for evaluating the accuracy and precision of other existing parametric and nonparametric methods used in practice. The other methods evaluated include those presented by Youd et al. (2001), Juang et al. (2003), Moss et al. (2006), Idriss and Boulanger (2008), Oommen et al. (2010), Rezania et al. (2011), and Yazdi et al. (2012).

Youd et al. (2001) presented the outcome of the 1995 and 1997 National Center for Earthquake Engineering Research (NCEER) workshops, summarizing the existing consensus deterministic methods. For CPT, Youd et al. (2001) presented the clean-sand threshold from Robertson and Wride (1998) for clean-sand conditions based on hand fit estimation. Juang et al. (2003) presented a probabilistic approach to determining the threshold utilizing artificial neural networks. Moss et al. (2006) presented an updated CPT-based case-history database and a probabilistic approach using a Bayesian framework. Idriss and Boulanger (2008) presented a deterministic approach using a modified database after Moss et al. (2006) and hand fit estimation of the threshold. Oommen et al. (2011) presented a probabilistic approach using maximum likelihood logistic regression (MLLR) that used a training and testing approach to verifying the threshold fit. Rezania et al. (2011) used evolutionary polynomial regression (EPR) in order to develop a deterministic threshold in three-dimensional (3D) space $\left(\mathrm{CSR}_{7.5}, q_{c 1 N}, \sigma_{v}^{\prime}\right)$. Yazdi et al. (2012) presented a deterministic threshold based on an adaptive neurofuzzy inference system (ANFIS), reducing the sampling bias by applying bootstrapping technique.

In the present study, a Bayesian framework was used for probabilistic analysis of liquefaction triggering threshold combined with a kernel density estimation (KDE) method that provides a nonparametric likelihood function. This means that no functional form is assumed since the derived likelihood from $\mathrm{KDE}$ is able to portray the intrinsic distribution of the liquefied/nonliquefied threshold. In other studies, the functional form of the triggering curve is either 
assumed or modeled with limited flexibility; here the triggering curve is free to be wholly dictated by the data. A number of metrics were then used to assess the performance of the resulting Bayes classifier that is the resulting form of the threshold. The optimum threshold of liquefaction was calculated with respect to the performance of Bayes classifier and smoothness of density function estimation. The metric scores of the proposed method, within what is often called a Confusion matrix, were compared with the other aforementioned methods.

\section{Liquefaction Triggering Framework}

The earthquake-induced cyclic stress ratio (CSR) at a given depth (z) within the soil profile is usually expressed as an average cyclic shear stress ratio, i.e.

$$
\mathrm{CSR}_{M, \sigma_{v}^{\prime}}=\frac{\tau_{\mathrm{avg}}}{\sigma_{v}^{\prime}}
$$

where $\tau_{\text {avg }}=$ average earthquake-induced shear stress; $\sigma_{v}^{\prime}=$ vertical effective stress; and the subscript CSR indicates that it is computed for a specific earthquake magnitude (moment magnitude, $M_{v}$ ) and the $\sigma_{v}^{\prime}$ at depth $z$. Seed and Idriss's (1971) simplified procedure calculates the CSR as

$$
\mathrm{CSR}_{M, \sigma_{v}^{\prime}}=0.65 \frac{\sigma_{v}}{\sigma_{v}^{\prime}} \frac{a_{\mathrm{max}}}{g} r_{d}
$$

where $\sigma_{v}=$ vertical total stress at depth $z ; a_{\max } / g=$ maximum horizontal acceleration (as a function of gravity) at the ground surface; and $r_{d}=$ shear stress reduction factor that accounts for the dynamic response of the soil profile. Duration of shaking impacts the soil's CRR and is computed by adjusting the earthquake-induced CSR to a reference $M=7.5$ and $\sigma_{v}^{\prime}=1 \mathrm{~atm}(101.25 \mathrm{kPa})$ for processing of a particular case histories, using the following expression:

$$
\mathrm{CSR}_{M=7.5, \sigma_{v}^{\prime}=1 \mathrm{~atm}}=0.65 \frac{\sigma_{v}}{\sigma_{v}^{\prime}} \frac{a_{\mathrm{max}}}{g} r_{d} \frac{1}{\operatorname{MSF}} \frac{1}{K_{\sigma}} \frac{1}{K_{\alpha}}
$$

where MSF = magnitude scaling factor, i.e., a proxy for duration or number of cycles of loading; $K_{\sigma}=$ correction factor for overburden effective stress; and $K_{\alpha}=$ correction factor for driving shear stress.

\section{Bayes Classifier}

The proposed method uses a Bayes classifier to distinguish between liquefaction and nonliquefaction occurrences. The Bayes classifier is a simple probabilistic classifier that has been utilized by previous researchers for probabilistic assessment of liquefaction (Juang et al. 2000, 2002). Considering $m$ number of classes $C=\left\{c_{1}, c_{2}, \ldots, c_{k}, \ldots, c_{m}\right\}$ within $n$ sample points of $X=\left\{x_{1}, x_{2}, \ldots, x_{n}\right\}$, the probability of event $c_{k}$, given event $X$, is written, $P\left(C=c_{k} \mid X\right)$ and is known as Bayes' theorem

$$
P\left(C_{k} \mid X\right)=\frac{P\left(c_{k}\right) P_{i}\left(x_{i} \mid C=c_{k}\right)}{\sum_{k=1}^{m} P\left(c_{k}\right) P_{i}\left(x_{i} \mid C=c_{k}\right)}
$$

where $P\left(c_{k}\right)=$ prior probability of occurrence of class $k$; and $P_{i}\left(x_{i} \mid C=c_{k}\right)=$ conditional probability of event $X$, given event $c_{k}$. Eq. (4) can be used for probability of liquefaction $\left(P_{L}\right)$ as follows:

$$
P_{L}=P(L \mid X)=\frac{P(L) P(X \mid L)}{P(L) P(X \mid L)+P(N L) P(X \mid N L)}
$$

where $P(L)$ and $P(N L)=$ prior probabilities of liquefaction and nonliquefaction, respectively; and $P(X \mid L)$ and $P(X \mid N L)=$ conditional probabilities or likelihood function for liquefied and nonliquefied class, respectively. Considering large number of samples for probability analysis and assuming no information about occurrence of liquefaction and nonliquefaction before prediction, $P_{L}$ largely relies on the likelihood function of liquefied and nonliquefied classes. In the next section, mathematical formulation of the $\mathrm{KDE}$ is discussed, which represent a nonparametric estimator of likelihood function for liquefied and nonliquefied classes.

\section{Kernel Density Estimation}

The likelihood functions can be written as $P_{i}\left(x_{i} \mid C=c_{k}\right)$, the probability that the feature value in the $i$ th position is equal to $x_{i}$ given class $c_{k}$. These were estimated using KDE from a set of labeled training data $(X, C) . \mathrm{KDE}$ is a nonparametric estimation of the probability density function population (Parzen 1962). KDE is a more-flexible estimator compared to the multinomial distribution, in the same way that KDE is considered more flexible than the histograms (Silverman 1986). So, instead of creating histograms with the attendant assumptions about bin width, and fitting theoretical distributions to these histograms with the attendant epistemic uncertainty from missing data and competing best-fit criteria, the KDE models the density more or less exactly using a variable smoothing function controlled by the bandwidth of the kernel. KDE can provide the accuracy of a variable bin-width histogram along with the flexibility of a theoretical distribution with unlimited number of parameters. The probability $P_{i}\left(x_{i} \mid C=c_{k}\right)$ was estimated using Eq. (6)

$$
P_{i}\left(x_{i} \mid C=c_{k}\right)=\frac{1}{N_{c} h} \sum_{j=1}^{N_{c}} K\left(x_{i}, x_{j|i| c_{k}}\right)
$$

where $N_{c}=$ number of the input data $X$ belonging to class $c_{k} ; x_{j|i| c_{k}}=$ future value in the $i$ th position of the $j$ th input $X=$ $\left\{x_{1}, x_{2}, \ldots, x_{n}\right\}$ in class $c_{k}$; and $h=$ window parameter or bandwidth. The bandwidth control the degree of smoothing applied to the density; $h_{x}$ controls the smoothness between conditional densities in the $x$-direction and $h_{y}$ controls the smoothness of each condition density in the $y$-direction. The kernel density estimator is a sum of bumps placed at the input data locations. The kernel function $K$ determines the shape of the bumps. The kernel role is spreading a probability mass of size $1 / N_{c}$ related to each data point in its neighborhood. A Gaussian kernel function was used in this study because of its mathematical utility, and can be written as

$$
K(x \mid \mu, \sigma)=\frac{1}{\sigma \sqrt{2 \pi}} e^{-(x-\mu)^{2} / 2 \sigma^{2}}
$$

where $\mu=$ mean; and $\sigma=$ width of Gaussian. In essence, the kernel is a nonlinear transformer $(\phi)$ for transforming data points from the input space (with dimension $D$ ) into a feature space having a higher dimension $L(L>D)$ in order to best-fit the data points (Fig. 1). The shape of the kernel function is not notably important, but, by contrast, the bandwidth matrix value is very important for density estimation (Wand and Jones 1995). The parameter $h$ determines the degree of smoothness of the density function. When $h$ is near to zero, a noisy estimation is gained by the undersmooth effect. When $h$ increases, the noise of estimation is reduced and density function approaches a more representative density, until it reaches an optimum value. With increasing $h$, after considering enough distance from optimum value, the estimation starts to lose details owing to the oversmooth effect. Therefore, to achieve an optimum probabilistic threshold, it is essential to find the optimum bandwidth 




Fig. 1. Mapping of dataset $\boldsymbol{X}$ by $\boldsymbol{\phi}$ into a higher dimensional space (reprinted from Yazdi et al. 2013, (C) ASCE)

considering smoothness of density function and performance of the Bayes classifier. In the present study, a set of metrics within a confusion matrix was used to evaluate the performance of the Bayes classifier. A brief explanation of confusion matrix is given in the following section.

\section{Performance Metrics}

A number of metrics were used to evaluate the performance of the proposed method and develop a quantitative basis for comparison with other methods. These are calculated using what is commonly called a confusion matrix (Fig. 2).

Suppose that occurrence of liquefaction is actually positive. Then

- True positive (TP) denotes the number of liquefied samples that were predicted correctly;

- True negative (TN) denotes the number of nonliquefied samples that were predicted correctly;

- False positive (FP) denotes the number of liquefied samples that were predicted incorrectly; and

- False negative (FN) denote the number of nonliquefied samples that were predicted incorrectly.

Based on the confusion matrix, the following metrics were used for evaluating and comparing prediction models:

$$
\begin{gathered}
\text { Sensitivity }=\frac{T P}{T P+F N} \\
\text { Specificity }=\frac{T N}{T N+F P} \\
\text { Accuracy }=\frac{T P+T N}{T P+F P+T N+F N} \\
\text { Precision }^{+}=\frac{T P}{T P+F P}
\end{gathered}
$$

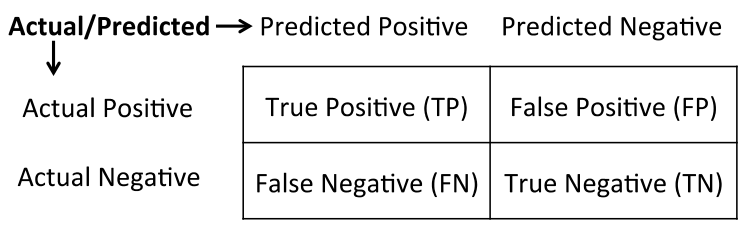

Fig. 2. Typical confusion matrix for a binary classification problem

$$
\begin{aligned}
& \text { Precision }^{-}=\frac{T N}{T N+F N} \\
& \mathrm{MCC}=\frac{T P \times T N-F N \times F P}{\sqrt{(T P+F N)(T N+F P)(T P+F P)(T N+F N)}} \\
& F \_ \text {measure }{ }^{+}=\frac{2 \times\left(\text { Precision }^{+}\right) \times \text {sensitvity }}{\text { Precision }+ \text { sensitvity }}
\end{aligned}
$$

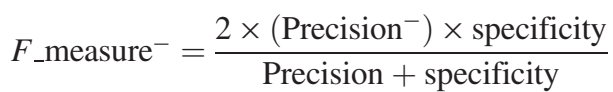

Accuracy (ACC) is known to be inappropriate for an imbalanced dataset since it becomes high when the liquefied samples in the majority class are favorably predicted. In this study, one of the standard measures used by statisticians is called the Matthews correlation coefficient (MCC; Matthews 1975). MCC indicates the degree of correlation between actual and predicted classes of the liquefied and nonliquefied. MCC values range is between 1, where all the predictions are correct, and -1 where none are correct. The MCC value is considered to be the best evaluation measure for the overall performance of a classifier method (Baldi et al. 2000). $F$-measure combines precision and sensitivity or specificity values to achieve a harmonic mean.

\section{CPT-Based Liquefaction Probabilistic Triggering}

Moss (2003) compiled a worldwide CPT database that includes 139 liquefied and 43 nonliquefied cases recorded from 18 different earthquakes spanning over 5 decades. The earthquakes included are 1964 Niigata, 1968 Inangahua, 1975 Haicheng, 1976 Tangshan, 1977 Vrancea, 1979 Imperial Valley, 1980 Mexicali, 1981 Westmorland, 1983 Nihonkai-Chubu, 1983 Borah Peak, 1987 Elmore Ranch, 1987 Superstition Hills, 1987 Edgecumbre, 1989 Loma Prieta, 1994 Northridge, 1995 Hyogoken-Nambu (Kobe), 1999 Kocaeli, and 1999 Chi-Chi earthquakes. Moss et al. (2004) added to the Imperial Valley case histories by using a modern electric cone at the Heber Road and River Park sites. Two liquefied case and seven nonliquefied case were added. In further research, Moss et al. (2011) conducted a field investigation to retest liquefaction and nonliquefaction sites from the 1976 Tangshan earthquake in China. Four liquefied cases were subsequently revised and nine cases added to the CPT database, including five liquefied and four nonliquefied cases. The present study includes all CPT data from these three studies, which then give 146 liquefied and 54 nonliquefied cases. The present analysis focuses on the clean-sand liquefaction threshold and avoids the nuanced and sometimes controversial 
issue of apparent fines content and how it influences the liquefaction threshold (Moss et al. 2006).

Liquefied and nonliquefied case data were separately fed into $\mathrm{KDE}$ function for modeling of the likelihood function. Since the bandwidth parameter $(h)$ affects the shape of likelihood function, the batches of $h_{x}$ and $h_{y}$ were optimized while respecting smoothness of likelihood and MCC value.

The larger the $h$ parameter, the smoother the overall fit. The optimum bandwidth was determined by treating the shape of the likelihood as a qualitative cost function and the MCC value as a quantitative cost function. Therefore, all of the possible combination of bandwidths from liquefied and nonliquefied cases were considered within a variation range of liquefaction probability from 0 to $100 \%$. The goal is a balance between the qualitative likelihood shape and the quantitative MCC value. The optimum bandwidth values were found to equal to $h=(0.63,0.07)$ for the liquefied data and $h=(0.82,0.07)$ for the nonliquefied data. Figs. 3 and 4 show the optimum likelihood function of liquefied and nonliquefied CPT data, respectively.

These two contour maps were combined into one representation of triggering. The probability of liquefaction $\left(P_{L}\right)$ was carried out according to Eq. (5). Contour map of probability of triggering of

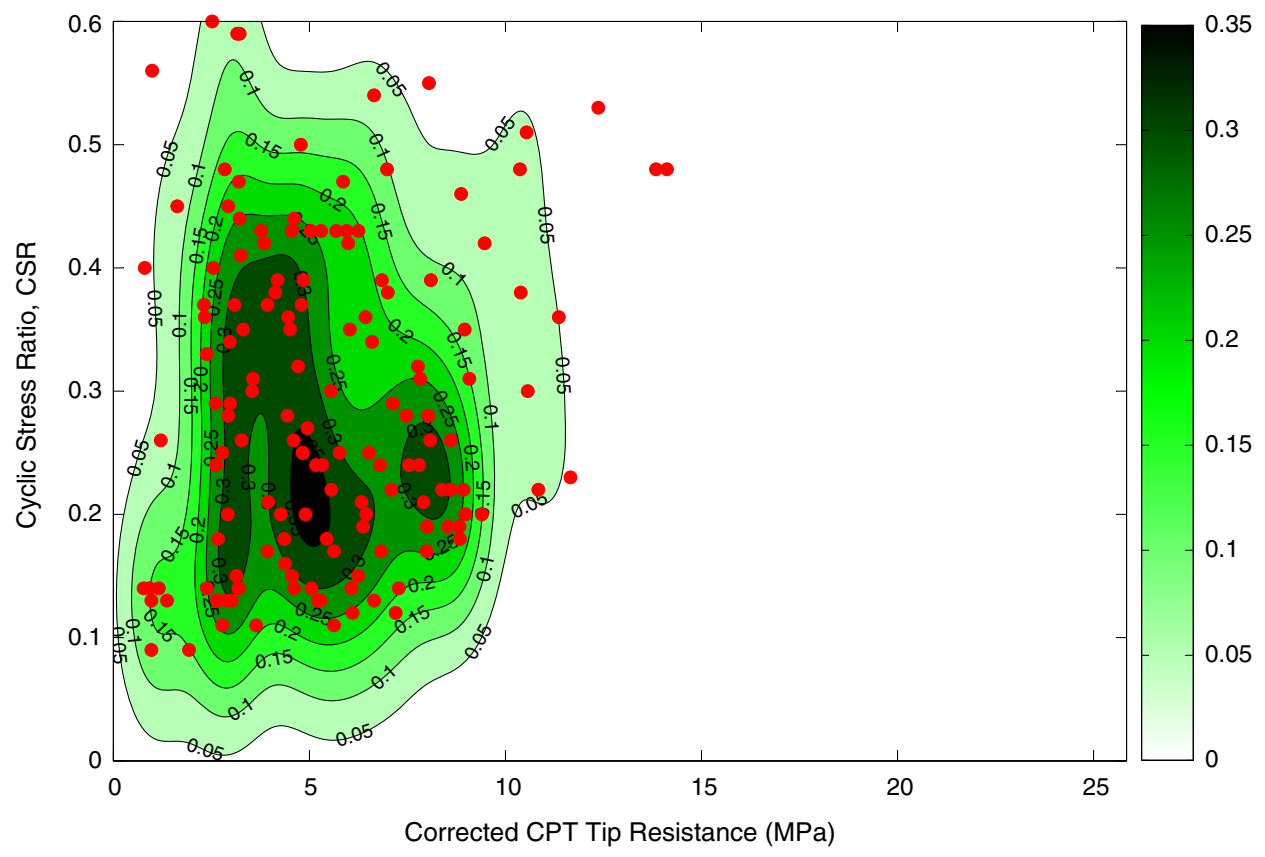

Fig. 3. Contour map of likelihood function of liquefied CPT data points with bandwidths from $h_{x}=0.5$ to $h_{x}=1$ and $h_{y}=0.05$ to $h_{y}=0.1$; solid dots are liquefied data points

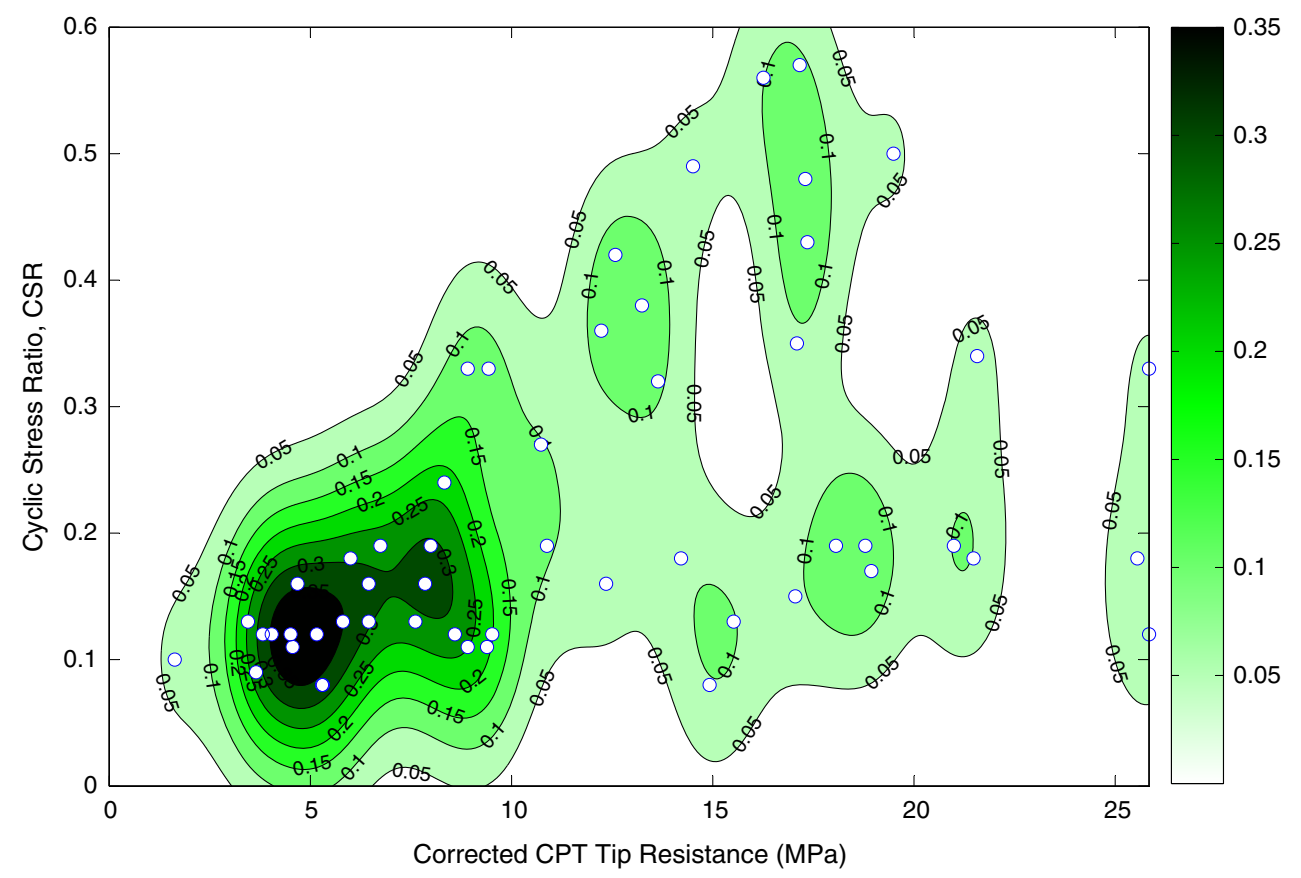

Fig. 4. Contour map of likelihood function for nonliquefied CPT data points with the same bandwidths as in Fig. 3; open circles are nonliquefied data points 


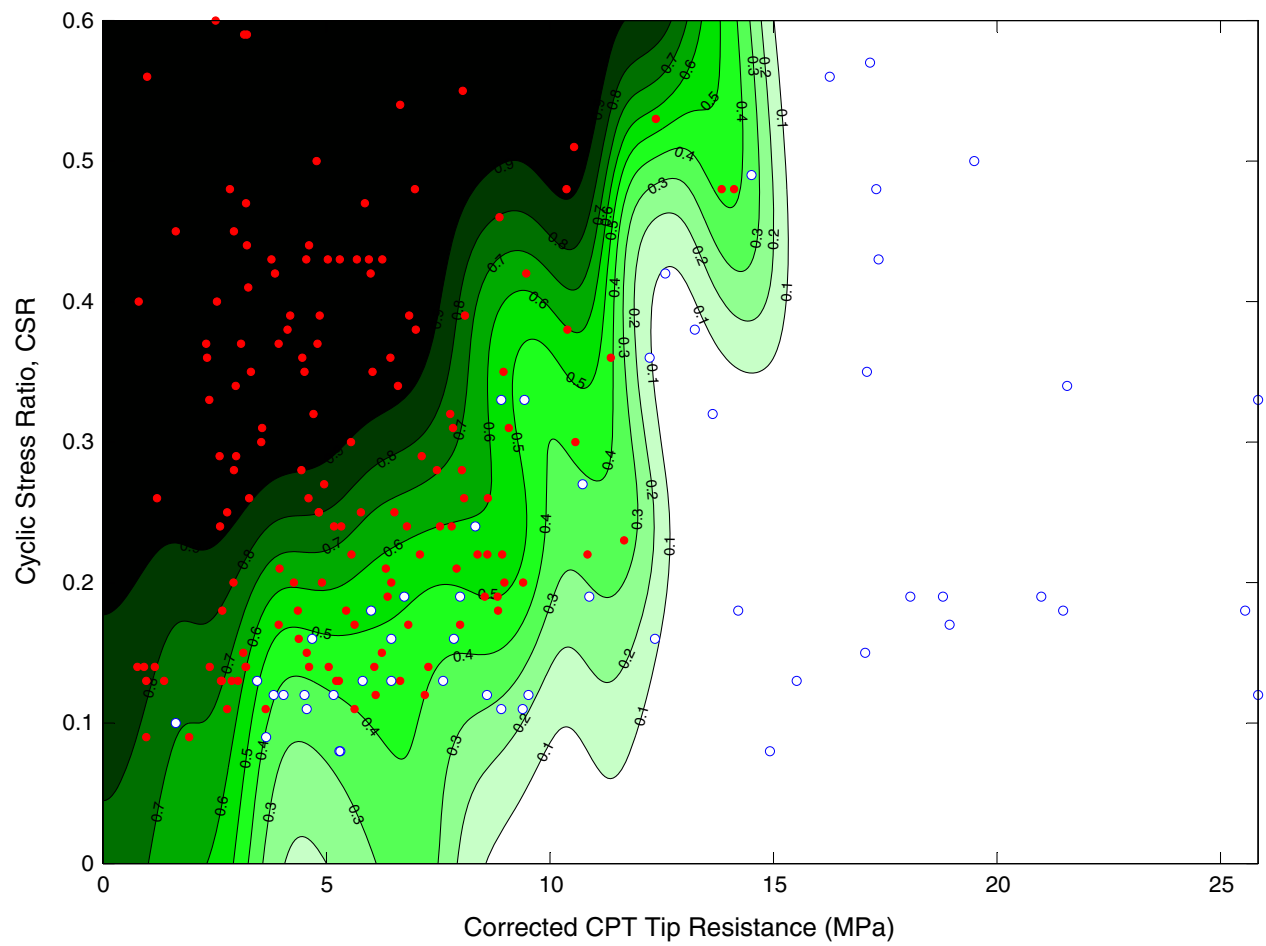

Fig. 5. Contour map of the probabilistic triggering threshold, combining what is shown in Figs. 3 and 4; solid dots are liquefied data, open circles are nonliquefied data; contour lines are shown for 0.1 increments of probability from 0.1 to 0.9

liquefaction covered from 0 to $100 \%$ of $P_{L}$ as a function of corrected CPT tip resistance and cyclic stress ratio presented in Fig. 5. The optimum threshold was determined based on the confusion matrix scores. A threshold of liquefaction probability of 0.4 was found to be the optimum classifier for this CPT database.

The performance of the proposed method was compared with seven other methods including those of Youd et al. (2001), Juang et al. (2003), Idriss and Boulanger (2008), Moss et al. (2006), Oommen et al. (2010), Rezania et al. (2011), and Yazdi et al. (2012). The confusion matrix scores were calculated based on CPT data from Moss et al. (2006) to achieve a reasonable comparison, respecting the literature assumptions (Yazdi et al. 2012) in the evaluation process. The confusion matrix scores for this proposed method and other methods from literature are tabulated in Table 1, where THL is liquefaction threshold and SVM is support vector machine. The results indicate a slightly higher performance of the Bayes classifier based on KDE as shown by the high values of the MCC, ACC, $F$-measure $^{+}$, and $F$-measure ${ }^{-}$. All methods, however, showed reasonable performance across the metrics, with no one method exhibiting superior performance over others. Regardless of which published method is used, it is recommended that the method should consider at least some portion of this uncertainty (Moss and Jacobs 2014). The rest of this study focuses on qualitatively interpreting the shape of the nonparametric triggering curve with respect to postliquefaction deformation characteristics to provide a quick screening tool for assessing liquefaction triggering consequences.

\section{Deformation Potential Based on the Proposed Liquefaction Trigger}

The unique shape of the nonparametric liquefaction triggering curve has some interesting characteristics that prompted a qualitative interpretation based on postliquefaction deformation behavior. Here, the nonparametric triggering curve is divided into three separate regions representative of different postliquefaction physics based on statistical ranges from prior studies (Fig. 6).

At the lowest values of penetration resistance, $q_{c 1}$ less than roughly $5 \mathrm{MPa}$, the nonparametric curve dives off, indicating that triggering is likely for very low levels of ground shaking and even no ground shaking. This region is dominated by very loose metastable soils and large deformations after triggering. Cases of static liquefaction have been observed in the past due to elevated

Table 1. Evaluation of Performance Measures of CPT Liquefaction Trigger

\begin{tabular}{|c|c|c|c|c|c|c|c|c|c|}
\hline Method & Details & $\mathrm{MCC}$ & ACC & Sensitivity & Specifically & Precision $^{+}$ & Precision $^{-}$ & $F$-measure $^{+}$ & $F$-measure $^{-}$ \\
\hline Youd et al. (2001) & - & 0.595 & 0.846 & 0.877 & 0.744 & 0.917 & 0.653 & 0.879 & 0.695 \\
\hline Juang et al. (2003) & $T H_{L}=0.45$ & 0.614 & 0.867 & 0.890 & 0.771 & 0.942 & 0.628 & 0.915 & 0.692 \\
\hline \multirow[t]{2}{*}{ Moss et al. (2006) } & $T H_{L}=0.15$ & 0.642 & 0.879 & 0.985 & 0.534 & 0.872 & 0.920 & 0.925 & 0.676 \\
\hline & $T H_{L}=0.50$ & 0.585 & 0.857 & 0.913 & 0.674 & 0.900 & 0.674 & 0.907 & 0.690 \\
\hline Idriss and Boulanger (2008) & - & 0.574 & 0.870 & 0.867 & 0.895 & 0.986 & 0.447 & 0.923 & 0.596 \\
\hline Oommen et al. (2010) & SVM & 0.675 & 0.890 & 0.978 & 0.604 & 0.888 & 0.896 & 0.931 & 0.722 \\
\hline Rezania et al. (2011) & EPR (3D space) & 0.576 & 0.841 & 0.878 & 0.721 & 0.910 & 0.646 & 0.894 & 0.681 \\
\hline Yazdi et al. (2012) & ANFIS $_{\text {upsample }}$ & 0.687 & 0.890 & 0.942 & 0.721 & 0.916 & 0.795 & 0.926 & 0.756 \\
\hline Proposed method & $T H_{L}=0.4$ & 0.779 & 0.923 & 0.919 & 0.939 & 0.986 & 0.721 & 0.951 & 0.816 \\
\hline
\end{tabular}

Note: Bold numbers indicate the highest value in each confusion matrix category. 


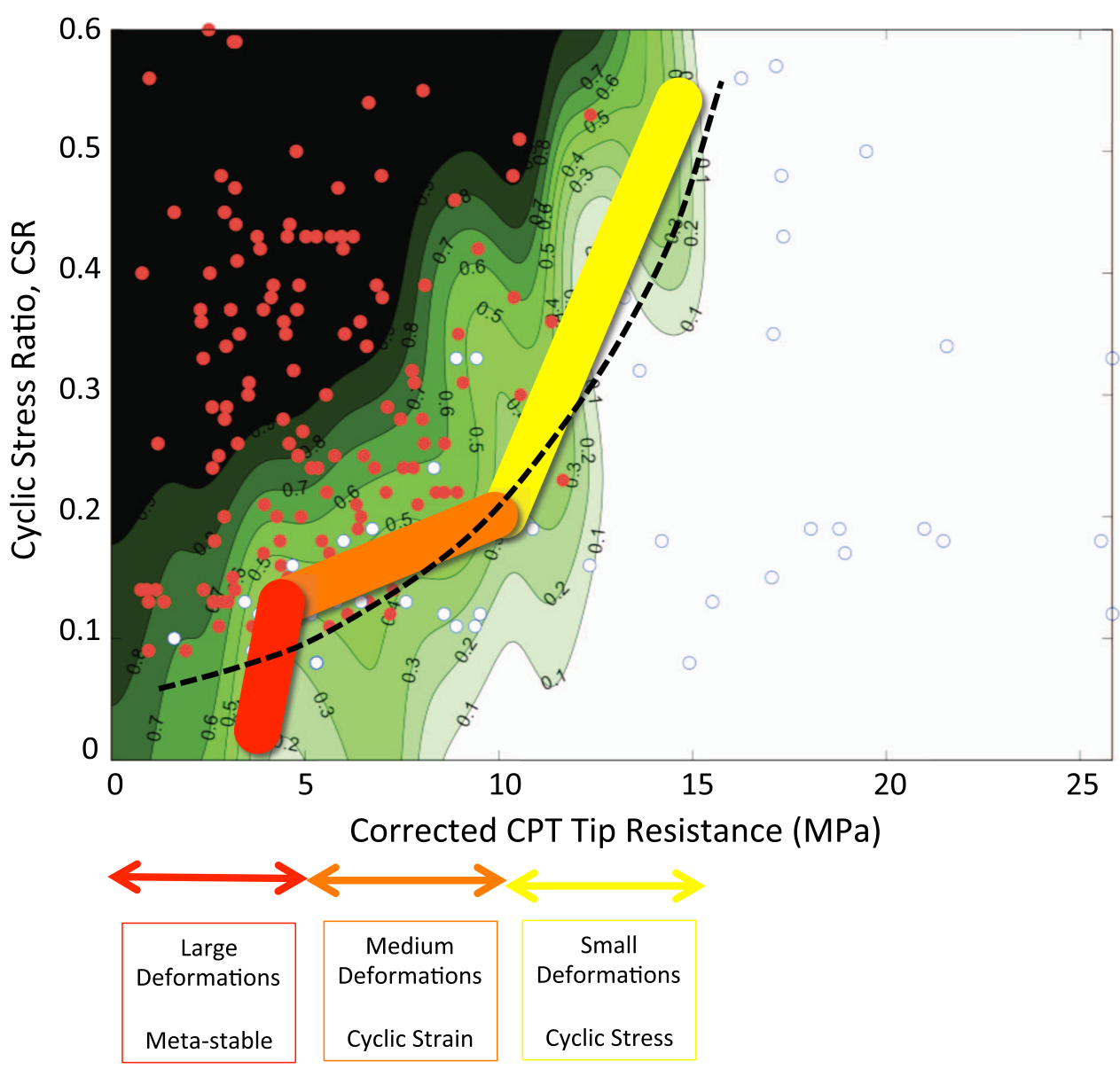

Fig. 6. Qualitative interpretation of nonparametric triggering curve for postliquefaction deformations; dotted line is the $P_{L}=20 \%$ triggering curve from Moss et al. (2006); the different ranges of penetration resistance based on prior studies are shown with respect to large, medium, and small deformations for conditions where there are no driving shear stresses; contour lines are shown for 0.1 increments of probability from 0.1 to 0.9

pore-pressure conditions or adverse static driving shear stresses (Casagrande 1976; Sladen et al. 1985). Deformations tend to be postseismic (or aseismic) in nature and continue after triggering due to the low residual strength $\left(s_{u, r}\right)$ of the soil with respect of the driving shear stresses $\left(\tau_{\text {static }}\right)$. Data from prior studies bounds this lower threshold of metastable behavior (Jefferies and Been 2006; Moss 2014).

The range of $q_{\mathrm{c} 1}$ between roughly 5 and $10 \mathrm{MPa}$ has been qualitatively interpreted as a second deformation region. Schneider and Moss (2011) showed that physics in this range are controlled by cyclic strain. In that study, cyclic strain theory and cyclic stress theory were combined to link two different physical phenomena that are typically represented together in the same liquefaction plot. The cyclic strain method was first put forward by Dobry et al. (1980) as an alternative to the commonly used cyclic stress method (Seed and Idriss 1971). By combining the cyclic strain and cyclic stress methods and evaluating the influence of shear stiffness, Schneider and Moss (2011) found a boundary between behaviors at roughly $10 \mathrm{MPa}$. This cyclic strain region is also in agreement with early liquefaction lab studies that found an inflected or concave down liquefaction triggering curve (Ishihara 1996) for the same loose to medium-dense range of saturated granular soils. Deformations in this range transition from large to medium and become more coseismic in nature as the penetration resistance increases (5-10 MPa). Data from the prior studies listed earlier provide support the upper threshold of $10 \mathrm{MPa}$ as a reasonable bound on this deformation region.
The third region is where $q_{c 1}$ is greater than roughly $10 \mathrm{MPa}$. In this region, the physics are controlled by cyclic stress as discussed in Schneider and Moss (2011). Deformations are often small in this range and are fully coseismic as a function of cycles of stress. These deformations can be exacerbated by static driving shear stresses, but soils at this density have limited capacity for deformation before locking up (Kammerer et al. 2004). These soils tend to deform only under high cyclic loading situations with adverse static stress conditions. Data from lab testing (e.g., Ishihara 1996; Wu et al. 2003; Kammerer et al. 2004) and limited reported deformations in the field database (Moss 2003) support this deformation range.

\section{Nominal Deformation Estimates}

Estimating deformations in practice is typically done using existing empirical methods; however, estimates are generally only accurate within an order of magnitude because of the complexity and uncertainty of the physics and inherent variability of the subsurface conditions. Generally a lower bound of expected postliquefaction deformations will occur when there are no driving shear stresses $\left(\tau_{\text {static }}=0\right)$. For site conditions where there are level ground conditions with no building-induced driving shear stresses and/or no free face conditions, the volumetric strains and shear strains can be estimated using the detailed lab work by Ishihara and Yoshimine (1992) or other similar studies (e.g., Shamoto et al. 1998; Wu et al. 2003; etc.). The Ishihara and Yoshimine work has been adopted here with some modifications to provide a nominal estimate of 
strains. The results were originally plotted as a function of the factor of safety against liquefaction $\left(F S_{L}=\mathrm{CRR} / \mathrm{CSR}\right)$, which has been transformed here into the probability of liquefaction $\left(P_{L}\right)$ by modeling CSR and CRR as lognormal distributions with a median coefficient of variation on the order of $28 \%$ based on the liquefaction database (Moss et al. 2006). The probability of liquefaction is

$$
P_{L}=\Phi(-\beta)
$$

where $\beta=$ reliability index, which can be estimated for lognormal CSR and CRR that are uncorrelated by (Rosenblueth and Esteva 1972; Moss 2013)

$$
\beta \simeq \frac{\ln \left(\frac{\mu_{\mathrm{CRR}}}{\mu_{\mathrm{CSR}}}\right)}{\delta_{\mathrm{CRR}}^{2}+\delta_{\mathrm{CSR}}^{2}}
$$

The modified figure (Fig. 7) shows solid curves of volumetric strain for different relative density values of the lab specimens and dashed curves of shear strain. The relative density has been statistically correlated to CPT tip resistance using the empirical equation for normally consolidated sands presented by Mayne et al. (2001)

$$
D_{R} \cong 100 \sqrt{\frac{q_{c 1}}{300}}
$$

Fig. 7 shows that volumetric strains up to $5 \%$ and shear strains up to $10 \%$ are possible in lab testing conditions. Because of the inherent boundary conditions, scaling issues, and other lab constraints, these strain estimates can be treated as a lower bound for estimating deformations for specific liquefiable layers in the field.

The practice of summing these strains over all layers that are potentially liquefiable, however, postulates that all potentially liquefiable layers that can liquefy will liquefy. Although multiple-layer

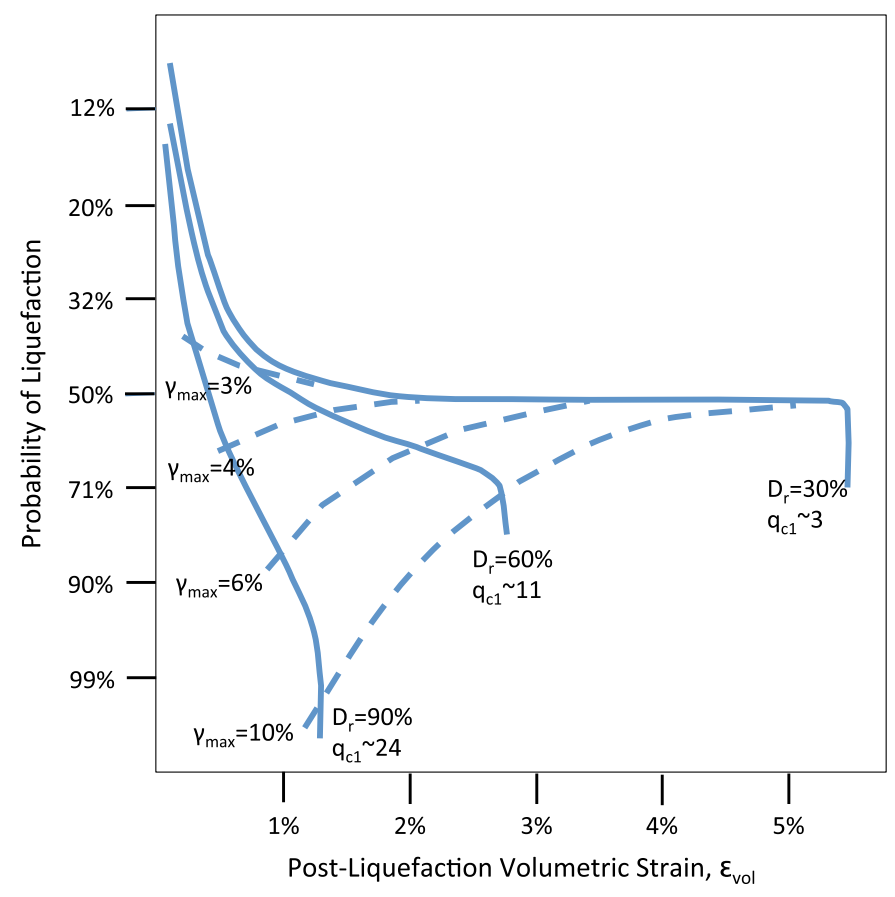

Fig. 7. Postliquefaction volumetric $\left(\varepsilon_{\mathrm{vol}}\right)$ and shear strain $\left(\gamma_{\max }\right)$ curves for different relative density $\left(D_{r}\right)$ and correlated cone penetration resistance $\left(q_{c 1}\right)$ of the lab samples; this figure is after Ishihara and Yoshimine (1992) where the $y$-axis has been transformed into probability of liquefaction liquefaction has been observed in the field (Youd and Bennett 1983), it is not a commonly reported occurrence and may be primarily due to pore-pressure migration from a weaker liquefied layer or very long duration shaking. An estimate of median deformations should use the weakest or critical layer (Moss et al. 2006) and strains from more layers only added to decrease the risk as warranted by project consequences.

\section{Deformations and Static Driving Shear Stresses}

When static driving shear stresses are present at a site due to sloping ground, free face conditions, or building/embankment stresses, the deformations are controlled by the relationship between the static driving shear stresses and the postliquefaction residual strength. Static driving shear stresses for an infinite slope condition (Park 2013) are the product of the vertical effective stress and the sine of the slope angle $\left(\tau_{\text {static }}=\sigma_{v}^{\prime} \sin \theta\right)$. These stresses are often normalized by the vertical effective stress to give alpha $\left(\alpha=\tau_{\text {static }} / \sigma_{v}^{\prime}\right)$. (Driving shear stresses can also affect the likelihood of liquefaction triggering, increasing the likelihood with lower penetration resistance, and decreasing the likelihood with higher penetration resistance, as captured with the $K_{\alpha}$ correction factor (e.g., Seed et al. 1985, 2003; Youd et al. 2001; Boulanger 2003).

Static driving shear stresses from a free face, embankment, or from a building footprint require elastic or numerical solutions to capture the two-dimensional effects. Elastic solutions for these conditions can be found in Poulos and Davis (1974) and other similar references. Cetin et al. (2012) considered foundation affects on deformations and presented a useful figure in that paper to help map static driving shear stress ratio.

When static driving shears stresses $\left(\tau_{\text {static }}\right)$ are higher than the liquefied undrained residual strength $\left(s_{u r}\right)$ (Seed et al. 2003), flow failure can occur with low levels of seismic loading (and in some cases no seismic loading). To quantify the range of penetration resistance for flow failures, empirical relationships of previous flow failure case histories were used (e.g., Seed and Harder 1985; Olson and Stark 2002; Kramer 2008; Weber 2015). The study by Olson and Stark (2002) presented the CPT penetration resistance of 33 flow failure case histories that were here statistically evaluated for the frequency of occurrence shown as a histogram in Fig. 8. The histogram indicates that the values of penetration resistance of the known database of flow failures range from 0 to $6 \mathrm{MPa}$ with a median value of $2.9 \mathrm{MPa}$. The frequency range and distribution correspond well with the region of the nonparametric triggering where it dives off. Deformations in this flow failure range can be unconstrained; once deformations are initiated, they often do not stop until the excess pore pressures are dissipated and the geometry of the flowing mass reaches a state where the driving forces (including momentum) are less than the new resisting forces. Flow failures as documented by Youd et al. (2002) have been observed on slopes steeper than $6 \%(\alpha \approx 0.06)$ and have exhibited deformations greater than $5 \mathrm{~m}$. Park (2013) reevaluated flow failure case histories and found the threshold of $3 \mathrm{~m}$ to be a dividing line between unconstrained flow and constrained lateral spreads. Whether greater than 3 or $5 \mathrm{~m}$, these unconstrained deformations are large and can cause significant damage to engineered features.

In these cases, the liquefied residual strength of the soil can be used in a limit equilibrium analysis to estimate the postliquefaction stability, and/or a calibrated nonlinear time domain finite-element/ finite-difference analysis can be used to approximate the postliquefaction deformations. Deformations can also be estimated using a lateral spreading-type model (e.g., Hamada et al. 1986; Youd et al. 2002). The term lateral spread generally defines a translational 

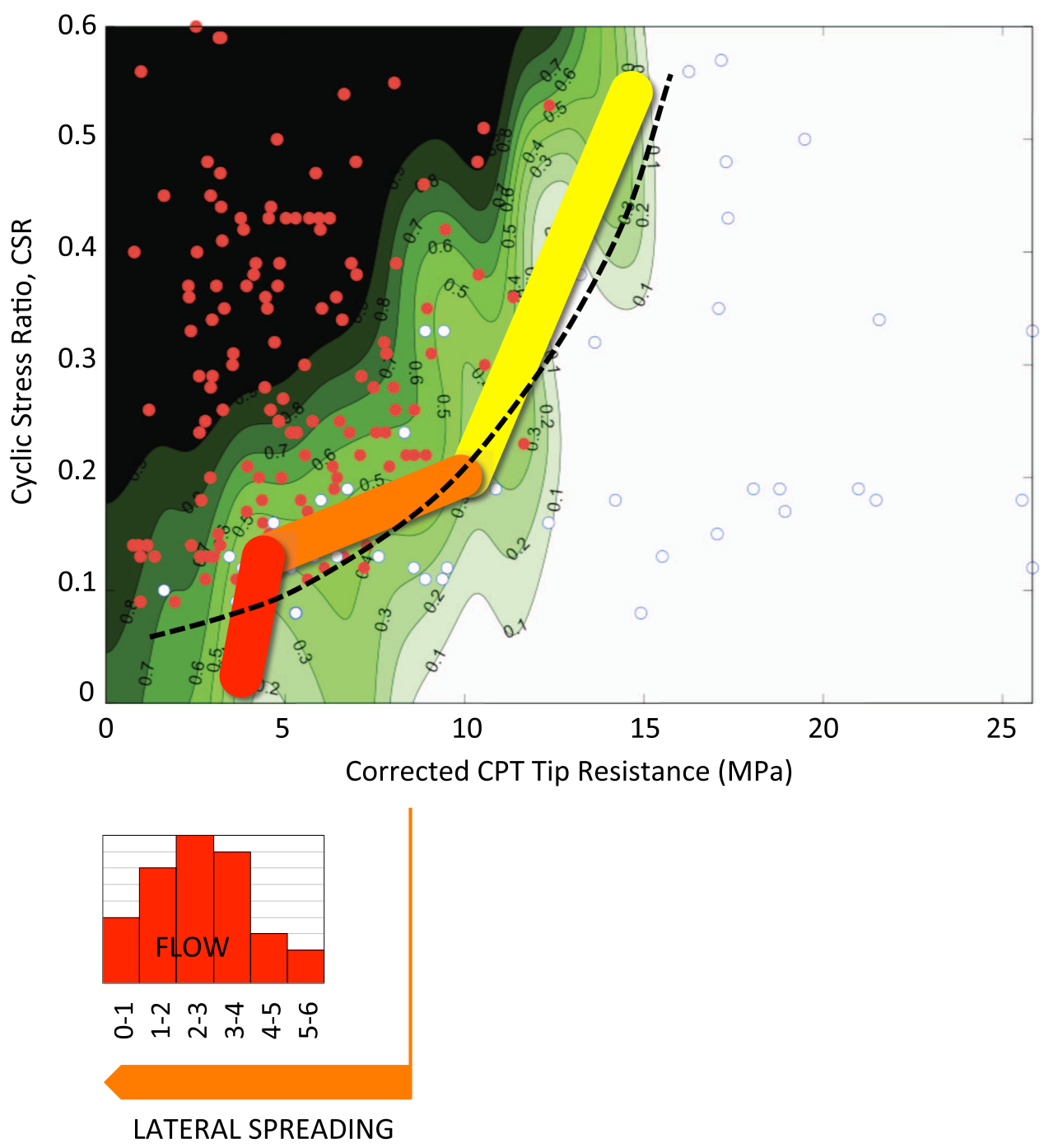

Fig. 8. Qualitative interpretation of nonparametric liquefaction triggering curve for postliquefaction deformations when driving shear stresses are present based on data from prior studies; contour lines are shown for 0.1 increments of probability from 0.1 to 0.9

failure due to a underlying liquefiable soil layer that is usually greater than $1 \mathrm{~m}$ thick (Youd et al. 2002), with soil response ranging from low penetration-resistance type flow failure $\left(\tau_{\text {static }}<s_{u, r}\right)$ to medium penetration-resistance type coseismic deformations $\left(\tau_{\text {static }} \geq s_{u, r}\right)$.

With increased tip resistance (3-8 MPa) and decreased driving shear stresses, reduced capacity for flow failure can be observed, but a site can experience medium deformations from static driving shear stresses. The empirical database of lateral spreading failures (Youd et al. 2002) shows that the range of penetration resistance is up to a corrected standard penetration test (SPT) blow count of 15 which is roughly equivalent to corrected cone tip resistance of $8 \mathrm{MPa}$ (Kulhawy and Mayne 1990). Here again, a lateral spreading model is a means of roughly estimating the deformation potential for sloping or free face conditions, and deformations are generally in the range of 5-0.5 m (Youd et al. 2002; Park 2013) for these conditions. Larger deformations can occur in this penetration resistance range if the excess pore pressures are trapped by an impermeable layer and/or void ratio redistribution results in a very low shear-resistance layer.

When confronted with driving shear stresses from buildings, embankments, or other features, deformation analysis can be more involved. Cetin et al. (2012) provided a basis for quick estimates of the order of magnitude deformations as a function of alpha.
For more-complex or high-consequence projects, a calibrated nonlinear time domain finite-element/finite-difference analysis can be used to approximate the postliquefaction deformations (Boulanger and Truman 1996; Kokusho 1999, 2003; Malvick et al. 2006; Park 2013).

For high penetration resistance above roughly 8-10 MPa, the soil can liquefy but the deformation potential is typically limited and case histories usually exhibit liquefaction effects such as small sand boils, limited ground cracking, and small volumetric/shear strains. Here, the expected deformations are generally less than $0.5 \mathrm{~m}$ and can be bounded by the modified Ishihara and Yoshimine plot (Fig. 7). More-detailed estimates in this range are rather difficult because the uncertainty often exceeds the magnitude of deformations and estimates are often biased. Hayati and Moss (2011) evaluated the small deformation range of $<0.2 \mathrm{~m}$ for level-ground sites with building-induced driving shear stresses. They found that common practice of summing lab-based volumetric strains (e.g., Ishihara and Yoshimine 1992) with depth often overestimates the measured postliquefaction volumetric deformations from field case histories, and the exclusion of nonliquefaction case histories results in a bias in the estimate. So in these specific situations, lab-based strains can provide an upper bound on the expected deformations. 


\section{Summary}

This study has reevaluated the liquefaction triggering threshold using a nonparametric approach. This approach avoids assumptions about the shape, form, and mathematics of the threshold and provides an unbiased fit to the existing CPT triggering database. A slightly improved fit over existing methods was found, and then this approach was used to evaluate the accuracy and precision of other methods in the literature. The results show that all the CPT triggering methods due a reasonably good job defining the clean-sand threshold, and certain methods have an edge over other methods depending on the metric used to quantify accuracy and precision.

The observed trends of the nonparametric triggering curve lent to a qualitative postliquefaction deformation interpretation based on ranges of penetration resistance from prior studies. Modified lab-based volumetric and shear strain curves (after Ishihara and Yoshimini 1992) were presented for estimating deformations where no driving shear stresses are present. The cone penetration ranges are: large deformations in the $0-5 \mathrm{MPa}$ range, medium deformations in the 5-10 MPa range, and small deformations for higher than $10 \mathrm{MPa}$. For a soil layer that is likely to liquefy, say a probability of liquefaction of $75 \%$, large deformations would be volumetric strains of roughly $3-5 \%$ and shear strains of roughly $8-10 \%$, Medium deformations would be volumetric strains of roughly $2-3 \%$ and shear strains of roughly $5-8 \%$. Small deformations would be volumetric strains less than roughly $2 \%$ and shears strains less than roughly $5 \%$. To frame this with an example, a 2-m-thick layer of saturated sandy soil with penetration resistance of $3 \mathrm{MPa}$ could exhibit up to $0.1 \mathrm{~m}$ of volumetric displacement and $0.2 \mathrm{~m}$ of shear displacement, whereas if the penetration resistance was $15 \mathrm{MPa}$, displacement would be unlikely but surely less than $0.03 \mathrm{~m}$ of volumetric and $0.08 \mathrm{~m}$ of shear displacement. In all of these cases, the deformations could be diminished before reaching the ground surface if there is sufficiently thick overlying nonliquefiable material (Ishihara 1985; Youd and Garris 1995).

When driving shear stresses are present (due to sloping ground, a free face, or building-induced stresses) then the deformations are controlled by postliquefaction residual strength with respect to the driving shear stress. Generally, for a penetration resistance less than $4 \mathrm{MPa}$ flow failure is the dominant failure mode, and for penetration resistance less than $8 \mathrm{MPa}$, lateral spreading is the dominant failure mode. Deformations from both of these failure modes are reasonably captured in existing lateral-spreading models (e.g., Youd et al. 2002). Flow failures typically result in lateral deformation greater than $3 \mathrm{~m}$, sometimes more than $5 \mathrm{~m}$, and can continue for longer runs depending on the conditions. Lateral spreads are more constrained and tend to exhibit deformations in the 0.5-3 $\mathrm{m}$ range. For penetration resistance values greater than 8-10 MPa with driving shear stresses present, deformations often have a volumetric less than $0.5 \mathrm{~m}$, but currently it is difficult to make estimates more precise than within approximately $\pm 0.2 \mathrm{~m}$.

\section{Conclusion}

The initial goal of this study was to examine the accuracy and precision of existing liquefaction triggering methods. In the process, a new nonparametric probabilistic triggering threshold has been developed. The new triggering threshold yielded interesting characteristics that readily lent themselves to a qualitative postliquefaction deformation interpretation. The range of cone penetration resistance values over which a liquefiable soil can exist has been divided into regions of large, medium, and small deformations and the influence of driving shear stresses is included in this interpretation.
As a whole, this study puts forward a simplified triggering and postliquefaction deformation screening tool that can be used for a quick assessment of the likelihood of liquefaction-induced deformations, and can be used to bound more-sophisticated liquefaction deformation analyses.

\section{References}

Baldi, P., Brunak, S., Chauvin, Y., Andersen, C. A. F., and Nielsen, H. (2000). "Assessing the accuracy of prediction algorithms for classification: An overview." Bioinformatics, 16(5), 412-424.

Boulanger, R. (2003). "Relating K $\alpha$ to relative state parameter index." J. Geotech. Geoenviron. Eng., 10.1061/(ASCE)1090-0241(2003)129: 8(770), 770-773.

Boulanger, R. W., and Truman, S. P. (1996). "Void redistribution in sand under post-earthquake loading." Can. Geotech. J., 33(5), 829-834.

Casagrande, A. (1976). "Liquefaction and cyclic deformation of sands: A critical review." Harvard soil mechanics series no. 88, Harvard Univ., Cambridge, MA.

Cetin, K. O., Unutmaz, B., and Jeremic, B. (2012). "Assessment of seismic soil liquefaction triggering beneath building foundation systems." Soil Dyn. Earthquake Eng., 43, 160-173.

Dobry, R., Powell, D. J., Yokel, F. Y., and Ladd, R. S. (1980). "Liquefaction potential of saturated sand-the stiffness method." 7th World Conf. on Earthquake Engineering, Vol. 3, ASCE, New York, 25-32.

Hamada, M., Yasuda, S., Isoyama, R., and Emoto, K. (1986). "Study on liquefaction induced permanent ground displacments." Association for the Development of Earthquake Prediction, 87.

Hayati, H., and Moss, R. E. S. (2011). "Evaluating bias of liquefactioninduced settlement methods for performance-based design." GeoRisk 2011: Geotechnical Risk Assessment and Management, ASCE, Reston, VA, 526-533.

Idriss, I., and Boulanger, R. (2008). "Soil liquefaction during earthquake." Earthquake Engineering Research Institute, Oakland, CA.

Ishihara, K. (1985). "Stability of natural deposits during earthquakes." Proc., 11th Int. Conf. on Soil Mechanics and Foundation Engineering, Vol. 1, Balkema, Rotterdam, Netherlands, 321-376.

Ishihara, K. (1996). Soil behaviour in earthquake geotechnics, Clarendon Press, Oxford University Press, Oxford, U.K.

Ishihara, K., and Yoshimine, M. (1992). "Evaluation of settlements in sand deposits following liquefaction during earthquakes." Soils Found., 32(1), 173-188.

Jefferies, M., and Been, K. (2006). Soil liquefaction: A critical state approach, Taylor \& Francis, New York.

Juang, C., Jiang, T., and Andrus, R. (2002). "Assessing probability-based methods for liquefaction potential evaluation." J. Geotech. Geoenviron. Eng., 10.1061/(ASCE)1090-0241(2002)128:7(580), 580-589.

Juang, C., Yuan, H., Lee, D., and Lin, P. (2003). "Simplified cone penetration test-based method for evaluating liquefaction resistance of soils." J. Geotech. Geoenviron. Eng., 10.1061/(ASCE)1090-0241(2003)129: $1(66), 66-80$.

Juang, C. H., Chen, C. J., Rosowsky, D. V., and Tang, W. H. (2000). "CPT-based liquefaction analysis-Part 2: Reliability for design." Géotechnique, 50(5), 593-599.

Kammerer, A. M., Seed, R. B., Wu, J., Riemer, M. F., and Pestana, J. M. (2004). "Pore pressure development in liquefiable soils under bidirectional loading conditions." Proc., 11th Int. Conf. on Soil Dynamics and Earthquake Engineering and 3rd Int. Conf. Earthquake Geotechnical Engineering, T. Nogami and R. B. Seed, eds., Vol. 2, Univ. of California, Berkeley, CA, 697-704.

Kokusho, T. (1999). "Water film in liquefied sand and its effect on lateral spread." J. Geotech. Geoenviron. Eng., 10.1061/(ASCE)1090-0241 (1999)125:10(817), 817-826.

Kokusho, T. (2003). "Current state of research on flow failure considering void redistribution in liquefied deposits." Soil Dyn. Earthquake Eng., 23(7), 585-603.

Kramer, S. L. (2008). "Evaluation of liquefaction hazards in Washington State." No. WA-RD 668.1, Washington State Dept. of Transportation, Office of Research and Library Services, Olympia, WA. 
Kulhawy, F. H., and Mayne, P. W. (1990). "Manual on estimating soil properties for foundation design." No. EPRI-EL-6800, Electric Power Research Institute, Palo Alto, CA.

Malvick, E. J., Kutter, B. L., Boulanger, R. W., and Kulasingam, R. (2006). "Shear localization due to liquefaction-induced void redistribution in a layered infinite slope." J. Geotech. Geoenviron. Eng., 10.1061/(ASCE) 1090-0241(2006)132:10(1293), 1293-1303.

Matthews, B. W. (1975). "Comparison of the predicted and observed secondary structure of T4 phage lysozyme." Biochimica et Biophysica Acta (BBA)-Protein Struct., 405(2), 442-451.

Mayne, P. W., Christopher, B. R., and DeJong, J. (2001). "Manual on subsurface investigations." National Highway Institute, Federal Highway Administration, Washington, DC.

Moss, R. E. S. (2003). "CPT-based probabilistic assessment of seismic soil liquefaction initiation." Ph.D. thesis, Univ. of California, Berkeley, CA.

Moss, R. E. S. (2013). Applied civil engineering risk analysis, Shedwick Press, San Luis Obispo, CA.

Moss, R. E. S. (2014). "A critical state framework for seismic soil liquefaction." Conf. Proc., CPT14, Las Vegas.

Moss, R. E. S., Collins, B., and Whang, D. H. (2004). "Retesting of liquefaction and non-liquefaction case histories in the imperial valley with the CPT." Earthquake Spectra, 21(1), 334-343.

Moss, R. E. S., and Jacobs, J. (2014). "Discussion of "Problems with liquefaction criteria and their applications in Australia" by R. Semple." Aust. Geomech. J., 15-34.

Moss, R. E. S., Kayen, R., Tong, L., Liu, S., Cai, G., and Wu, J. (2011). "Retesting of liquefaction and nonliquefaction case histories from the 1976 Tangshan earthquake." J. Geotech. Geoenviron. Eng., 10.1061/ (ASCE)GT.1943-5606.0000406, 334-343.

Moss, R. E. S., Seed, R., Kayen, R., Stewart, J., Der Kiureghian, A., and Cetin, K. (2006). "CPT-based probabilistic and deterministic assessment of in situ seismic soil liquefaction potential." J. Geotech. Geoenviron. Eng., 10.1061/(ASCE)1090-0241(2006)132:8(1032), 1032-1051.

Olson, S. M., and Stark, T. D. (2002). "Liquefied strength ratio from liquefaction flow failure case histories." Can. Geotech. J., 39(3), 629-647.

Oommen, T., Baise, L., and Vogel, R. (2010). "Validation and application of empirical liquefaction models." J. Geotech. Geoenviron. Eng., 10.1061/(ASCE)GT.1943-5606.0000395, 1618-1633.

Oommen, T., Baise, L., and Vogel, R. (2011). "Sampling bias and class imbalance in maximum-likelihood logistic regression." Math. Geosci., 43(1), 99-120.

Park, D. S. (2013). "Analyzing liquefaction induced instability and deformation of slopes using static shear stress and residual strength." ASCE Geo-Congress 2013 Proc., ASCE, Reston, VA, 854-863.

Parzen, E. (1962). "On estimation of a probability density function and mode." Ann. Math. Stat., 33(3), 1065-1076.

Poulos, H. G., and Davis, E. H. (1974). Elastic solutions for soil and rock mechanics, Wiley, New York.

Rezania, M., Faramarzi, A., and Javadi, A. A. (2011). "An evolutionary based approach for assessment of earthquake-induced soil liquefaction and lateral displacement." Eng. Appl. Artif. Intell., 24(1), 142-153.

Robertson, P. K., and Wride, C. E. (1998). "Evaluating cyclic liquefaction potential using the cone penetration test." Can. Geotech. J., 35(3), 442-459.
Rosenblueth, E., and Esteva, L. (1972). "Reliability basis for some Mexican codes." Spec. Publ., 31, 1-42.

Schneider, J. A., and Moss, R. E. S. (2011). "Linking cyclic stress and cyclic strain based methods for assessment of cyclic liquefaction triggering in sands." Geotech. Lett., 1(2), 31-36.

Seed, H. B., and Idriss, I. M. (1971). "Simplified procedure for evaluating soil liquefaction potential." J. Soil Mech. Found. Div., 97(9), 1249-1273.

Seed, H. B., Tokimatsu, K., Harder, L. F., and Chung, R. M. (1985). "Influence of SPT procedures in soil liquefaction resistance evaluations." J. Geotech. Eng., 10.1061/(ASCE)0733-9410(1985)111: 12(1425), 1425-1445.

Seed, R. B., et al. (2003). "Recent advances in soil liquefaction engineering: A unified and consistent framework." Proc., 26th Annual ASCE Los Angeles Geotechnical Spring Seminar, Long Beach, CA.

Seed, R. B., and Harder, L. F. (1985). "SPT-based analysis of cyclic pore pressure generation and undrained residual strength." Proc., H. Bolton Seed Memorial Symp., Vol. 2, BiTech Publishers, Vancouver, BC, Canada, 351-376.

Shamoto, Y., Zhang, J. M., and Tokimatsu, K. (1998). "Methods for evaluating residual post-liquefaction ground settlement and horizontal displacement." Soils Found., 38, 69-84.

Silverman, B. W. (1986). Density estimation for statistics and data analysis, Chapman and Hall, London.

Sladen, J. A., D'Hollander, R. D., Krahn, J., and Mitchell, D. E. (1985). "Back analysis of the Nerlerk berm liquefaction slides." Can. Geotech. J., 22(4), 579-588.

Wand, M. P., and Jones, M. C. (1995). "Monographs on statistics and applied probability." Kernel smoothing, Chapman and Hall, London, U.K.

Weber, J. P., et al. (2015). Engineering evaluation of post-liquefaction strength, Univ. of California, Berkeley, CA.

Wu, J., Seed, R. B., and Pestana, J. M. (2003). "Liquefaction triggering and post liquefaction deformations of monterey $0 / 30$ sand under unidirectional cyclic simple shear loading." Geotechnical Engineering Rep. No. UCB/GE-2003/01, Univ. of California, Berkeley, CA.

Yazdi, J., Kalantary, F., and Yazdi, H. (2012). "Prediction of liquefaction potential based on CPT up-sampling." Comput. Geosci., 44(0), $10-23$.

Yazdi, J., Kalantary, F., and Yazdi, H. (2013). "Prediction of elastic modulus of concrete using support vector committee method." J. Mater. Civ. Eng., 10.1061/(ASCE)MT.1943-5533.0000507, 9-20.

Youd, T., et al. (2001). "Liquefaction resistance of soils: Summary report from the 1996 NCEER and 1998 NCEER/NSF workshops on evaluation of liquefaction resistance of soils." J. Geotech. Geoenviron. Eng., 10.1061/(ASCE)1090-0241(2001)127:10(817), 817-833.

Youd, T. L., and Bennett, M. J. (1983). "Liquefaction sites, Imperial Valley, California." J. Geotech. Eng., 10.1061/(ASCE)0733-9410(1983)109: 3(440), 440-457.

Youd, T. L., and Garris, C. T. (1995). "Liquefaction-induced groundsurface disruption." J. Geotech. Eng., 10.1061/(ASCE)0733-9410 (1995)121:11(805), 805-809.

Youd, T. L., Hansen, C. M., and Bartlett, S. F. (2002). "Revised multilinear regression equations for prediction of lateral spread displacement." J. Geotech. Geoenviron. Eng., 10.1061/(ASCE)1090-0241(2002)128: 12(1007), 1007-1017. 